\title{
Multi-scale CAFE Modelling for Hot Deformation of Aluminium Alloys
}

\author{
M.F. Abbod ${ }^{1}$, I.C. Howard ${ }^{3}$, D.A. Linkens ${ }^{2}$, and M. Mahfouf ${ }^{2}$
}

IMMPETUS

Institute for Microstructural and Mechanical Process Engineering, The University of Sheffield

${ }^{1}$ School of Engineering and Design, Brunel University, Uxbridge UB8 3PH, UK

${ }^{2}$ Department of Automatic Control and Systems Engineering,

${ }^{3}$ Department of Mechanical Engineering

University of Sheffield, Sheffield S1 3JD, UK

Maysam.Abbod@brunel.ac.uk

\begin{abstract}
The multi-Scale CAFE modelling system utilises Cellular Automata, Finite Elements and a Hybrid Modelling technique which combines neurofuzzy models and physical equations to simulate hot deformation of $\mathrm{Al}-1 \% \mathrm{Mg}$ aluminium alloys using the commercial finite element software package ABAQUS $^{\mathrm{TM}}$. This paper addresses the issue of capturing microstructural details and providing macro linkage by simulating two phenomena. The first defines a suitable length scale such that numerical models are sufficient in detail and are appropriate in terms of computational time. The second is the feasibility using Cellular Automata (CA) as an additional technique that can be used in conjunction with a conventional Finite Elements (FE) representation to model material heterogeneity and related properties. This is done by identifying an abstract scale in between the micro and macro scales, termed the "mesoscale" to obtain a multi-scale CAFE modelling technique that utilises the CA technique to represent initial and evolving microstructural features at an appropriate length obtained using an overlying FE mesh.
\end{abstract}

\section{Introduction}

During hot deformation of aluminium alloys, rolling will change particularly the deformation texture and recrystallisation texture. Since aluminium and its alloys have high stacking-fault energy, hot deformation will not provide mechanical twining. Incorporating microstructural details in thermomechanical processing models is well recognised by many researchers [4], [6].

Finite elements (FE) method can be used with numerical formulations that describe the behaviour of different material models to elicit the response of a structure to strain. The material microstructure model can be expressed mathematically by a implicit semi-empirical methodology which is based on the use of trigonometric functions that relate a change in equivalent strain to a change of equivalent stress. FE models based on this approach [3] are successful in predicting the macro behaviour of metal flow stress and load, but fail to model the evolution of the underlying microstructure. In addition, constants used in these formulations should be employed with caution outside the conditions beyond which they have been tested. 
The microstructural evolution and its effects during the rolling process can be modelled using physically-based models. These relationships relate the evolution of the microstructal variable to a change in the equivalent strain [5]. Conventional FE models based on this approach use a statistically-averaged value to represent the evolution of an internal variable that is linked to each finite element [2]. The local variations, either present initially or evolving during the process itself, can be monitored individually.

An alternative method is to model the microstructure explicitly. This methodology requires the reduction of the size of each finite element to the size of a single grain or even lower which computationally extensive. This paper introduces a novel framework which couples hybrid modelling of thermomechanical processing [7], the mathematical tool of Cellular Automata within the FE code to form a Cellular Automata-based Finite Element (CAFE) model. Results for applications of the augmented CAFE model as applicable to hot deformation are presented.

\section{The Multi-scale Microstructure CAFE Model}

The CAFE model [1] is a framework to capture different strata of initial and evolving microstructures during deformation through a materials-mechanics formulism. CAFE is based on three steps: 1) approximation of relevant microstructure, 2) distribution of macro variables on the meso domain and 3) averaging meso-variables to the macro domain.

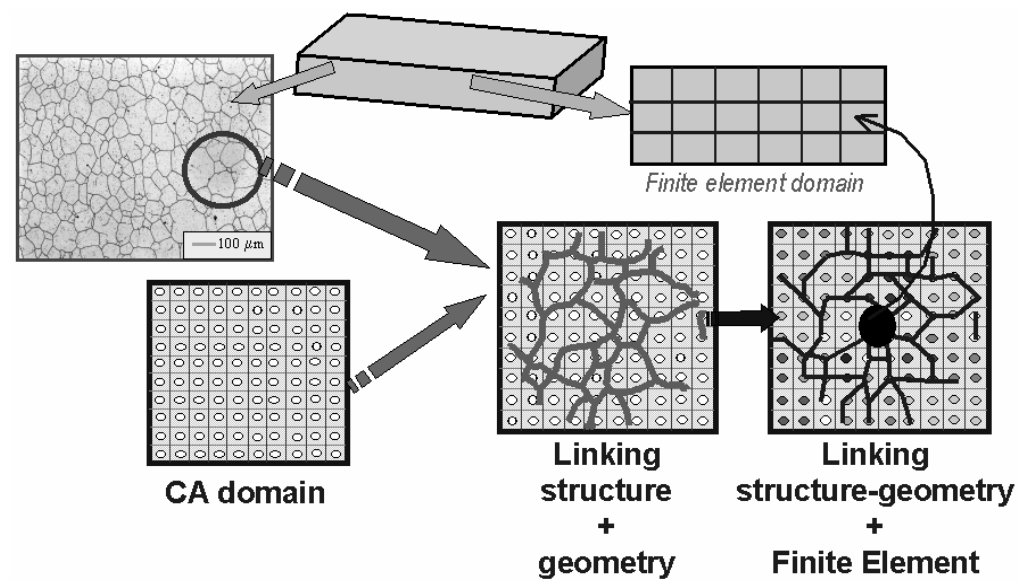

Fig. 1. Linking Structure and Geometry to incorporate microstructural details into geometric space of the Finite Element domain

CAFE is based on the structure shown in Fig. 1 which illustrates the methodology of linking the microstructure to the Finite Element domain using CA. The material slab under investigation is discretised into 18 rectangular finite elements. Each finite element cell simulates the microstructure of aluminium alloys and comprises equiaxed grains of the order of $80 \sim 100$ microns. The spatial information is mapped 
onto the CA domain, the size of which is determined by the size of each CA cell (marked by open circles). Finally, the CA domain is linked to the integration point (black circle in figure) of each finite element. The next step in the CAFE methodology is to transfer the independent macro level variables into the meso level CA variables. These macro variables are supplied by the overlying FE either at its nodes, centroids or Gaussian integration points.

To start the simulation of a microstructure, grain nuclei are randomly scattered over the domain. A second-level array of CA cells of $0.1 \sim 5 \mu \mathrm{m}$ size represent these nuclei. All nuclei can be assumed to be present at the start of the simulation as is the case with site-saturated nucleation or they can continue to appear during the simulation as a function of the deformation variables. The present work employs the first approach and uses a random function to generate and locate these nuclei on the domain. All first-level CA cells except those occupied by the nuclei have a "zero" orientation that represents the matrix within which the grain can grow. The presence or absence of nuclei is an internal variable for the first-level CA cell. Other internal variables are the spatial location of the $\mathrm{CA}$ cell and the orientation of the microstructure it will represent. This form of linking a microstructural entity to a spatial geometric entity allows embedding different structure-geometry relationships in a multi-level CA.

In the initial simulation validation stage, a Plane Strain Compression (PSC) model was simulated. Both the specimen and tool are modelled using 4-noded quadrilateral elements. All the stock elements are initialised with a starting temperature of $400^{\circ} \mathrm{C}$ and the tool elements are at $390^{\circ} \mathrm{C}$. Friction at the tool-stock interface is modelled using the Amonton-Coulomb law with a constant coefficient of friction of 0.1 . The heat transfer coefficient at the interface is $80 \mathrm{~kW} / \mathrm{m}^{2} \mathrm{~K}$. The instantaneous tool velocity changes with the instantaneous specimen height and is controlled to achieve a constant nominal strain-rate of $3 \mathrm{~s}^{-1}$. The stock is thickness-reduced by $41 \%$ during the deformation. The simulation begins with the introduction of a representative microstructure into each finite element. Results for the von-Mises stress and the strain are shown in Fig. 2 which gives the advantages of showing the microstructure of the material as well as the grains structure.

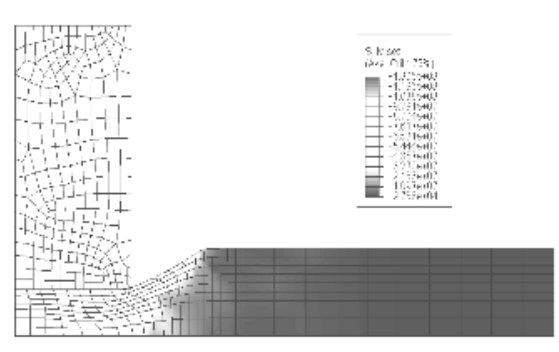

(a) von Mises stress

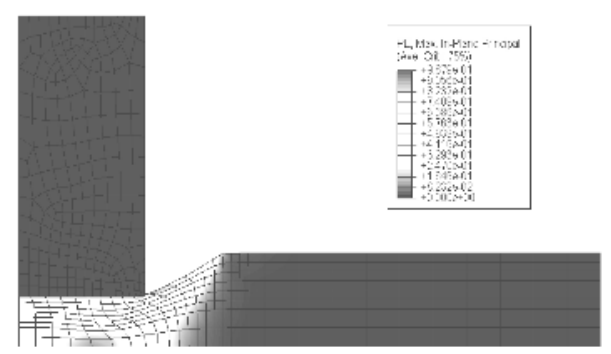

(b) Equivalent strain

Fig. 2. The multi-scale CAFE simulation for PSC test of commercial purity aluminium alloy, $\mathrm{T}_{\text {strip }}=400^{\circ} \mathrm{C}, \mathrm{T}_{\text {tool }}=390^{\circ} \mathrm{C}$ 


\section{Conclusions}

This paper presents a generic method for modelling the microstructure within the continuum formulation of finite element structural modelling. The Multi-Scale CAFE framework was applied to model the behaviour of the materials during hot deformation using a hybrid modelling technique. The model has simulated the microstructure of the material during hot deformation. The material behaviour was developed from the physically-based relations that relate the stress evolution to the total dislocation density using the CAFE approach. Recrystallisation behaviour was modelled using a critical dislocation density and subgrain size at a CA level.

\section{Acknowledgements}

The authors gratefully acknowledge the UK EPSRC (Engineering and Physical Sciences Research Council) for their financial support under grant number GR/R70514/01.

\section{References}

1. Beynon, J.H., Das, S., Howard, I.C., Palmiere, E.J., Chterenlikht, A.: The Combination of Cellular Automata and Finite Elements for the Study of Fracture: The CAFE Model of Fracture. $14^{\text {th }}$ European Conference on Fracture - ECF 14, Cracow, Poland (2002).

2. Busso, E.P.: A Continuum Theory for Dynamic Recrystallisation with Icrostructure-Related Length Scales. International Journal of Plasticity, Vol. 14, No. 4-5 (1998) 319-353.

3. Dutta, K.: Finite Element Modelling of Hot Rolling. PhD Thesis. The University of Sheffield (1996).

4. Jonas, J.J., Sellars, C.M., McG Tegart, W.J.: Strength and Structure Under Hot Working Conditions. Metallurgical Reviews, Vol. 130 (1969) 1-24.

5. Marthinsen, K., Nes, E.: A General Model for Metal Plasticity. Material Science and Engineering. Vol. A234-236 (1997) 1095-1098.

6. Sellars, C.M., Whiteman, J.A.: Recrystallisation and Grain Growth in Hot Rolling. Metal Science, (1979) 187-194.

7. Zhu, Q., Abbod, M.F., Talamantes-Silva, J., Sellars, C.M., Linkens, D.A., Beynon, J.H.: Hybrid Modelling of Aluminium-Magnesium Alloys During Thermomechanical Processing in Terms of Physically-Based, Neuro-Fuzzy and Finite Elements Models. Acta Mat, Vol. 51 (2003) 5051-5062. 